\title{
Effect of Time on the Adsorption of Methylene Blue, Methyl Orange and Indigo Carmine onto Activated Carbon
}

\author{
Ocholi O.J ${ }^{1}$, Gimba C.E ${ }^{1}$., Ndukwe G.I ${ }^{1}$., Turoti $\mathrm{M}^{2}$., \\ Abechi S.E ${ }^{1}$ and Edogbanya P.R.O ${ }^{3}$ \\ ${ }^{1}$ Department of Chemistry, Ahmadu Bello University, Zaria- Nigeria. \\ ${ }^{2}$ Department of Chemistry, Obafemi Awolowo University, Ile Ife- Nigeria. \\ ${ }^{3}$ Department of Biological Sciences, Ahmadu Bello University, Zaria- Nigeria.
}

\begin{abstract}
The effect of time on the adsorption of methylene blue (MB), methyl orange $(M O)$ and indigo carmine(IG) from aqueous media onto activated carbon (AC) was monitored using two(2)similar experimental procedures. In the first procedure, $20 \mathrm{mg}$ of $A C$ was interacted separately with $10 \mathrm{~cm}^{3}$ of $20 \mathrm{ppm}$ of each dye at residence time of $5,10,15,20,30,45$ and 60 minutes, while in the second procedure, 10mg of AC was interacted separately with $10 \mathrm{~cm}^{3}$ of 10ppm at residence time of 15, 30, 45 and 60 minutes respectively. From the results, $M O$ was adsorbed more than the others while MB was the most time dependent of the three dyes. Results also showed that chemisorption was the predominant adsorption type, while the adsorption process was particle-diffusion controlled.
\end{abstract}

Keywords: Adsorption, Methylene blue, Methyl orange, Indigocarmine.

\section{Introduction}

Time is crucial to the success of most chemical processes. For Adsorption processes, the rates of adsorption or the time taken to reach equilibrium is usually monitored; where fast rates are usually desired. Fast adsorption rates are related to high degree of affinity between the adsorbate and adsorbent via chemisorption. (Hameed et al. in Kathiresan et al., 2010.)

The rate or kinetics of adsorption processes can give valuable information regarding the "order" of the process. It can help to predict if the process is Physisorption or Chemisorption, and it can also give insight to the sorption process( i.e whether film diffusion or particle diffusion is occuring)(Horsfall Jnr and Spiff, 2005). For this work, The effect of time on the adsorption of individual dyes(MB, MO and IG) was monitored as part of a preliminary work which was to study the adsorption of multi-component adsorbate systems onto activated carbon surfaces.

\section{Activated Carbon Sample}

\section{Materials And Methods}

The activated carbon sample used for the work was extra pure food grade granular activated carbon from Merck Darmstadt Germany. Below is the summary of its factory specifications. The sample was pulverized and sieved. Particle size of between $425 \mu \mathrm{m}-850 \mu \mathrm{m}$ was used for the work.

Table 1: Factory Specifications of AC sample

\begin{tabular}{|l|l|}
\hline Identity & Conforms \\
\hline Particle Size & $1.5 \mathrm{~mm}$ \\
\hline Substances soluble in Nitric acid & $\leq 5 \%$ \\
\hline Chlorides & $\leq 500 \mathrm{ppm}$ \\
\hline Cyano compounds & Passes test \\
\hline Arsenic & $\leq 5 \mathrm{ppm}$ \\
\hline Iron & $\leq 500 \mathrm{ppm}$ \\
\hline Lead & $\leq 20 \mathrm{ppm}$ \\
\hline Zinc & $\leq 100 \mathrm{ppm}$ \\
\hline n-hexane adsorption & $\geq 30 \%$ \\
\hline Residue on ignition & $\leq 6 \%$ \\
\hline Loss on drying & $\leq 10 \%$ \\
\hline
\end{tabular}

Preparation of methylene blue, methyl orange and indigocarmine stock solutions

$1000 \mathrm{ppm}$ solutions of each dye was prepared by separately weighing $0.5 \mathrm{~g}$ of $\mathrm{MO}$ and $\mathrm{MB}$ dye and were partially dissolved in a beaker using distilled water. It was then transferred into a $500 \mathrm{~cm}^{3}$ volumetric flask and was made up to the mark using distilled water. They were then stored in amber coloured bottles and kept in a locker. For IG, smaller volumes of stock solution were prepared (i.e. $100 \mathrm{~cm}^{3}$ capacity) when needed. This was 
because it is not stable on storage. It goes from blue to orange colour, hence the need to freshly prepare it when needed. The various needed concentrations of the dyes were then prepared via serial dilution.

\section{Dye (adsorbate) molecules.}

Three different dye molecules were used as adsorbates in this work. They are: Methylene Blue(MB), MethylOrange(MO) and Indigo Carmine(IG).

\section{Methylene blue(MB)}

Methylene blue(CI 52015); Methylthionine Chloride; 3,7-bis[Dimethylamino]-Phenazothionium Chloride(SIGMA , 1991), is a potent Cationic dye with chemical formular $\mathrm{C}_{16} \mathrm{H}_{18} \mathrm{ClN}_{3} \mathrm{~S}$ and formular weight of $319.9 \mathrm{~g} / \mathrm{mol}$. Below is the structural formular.

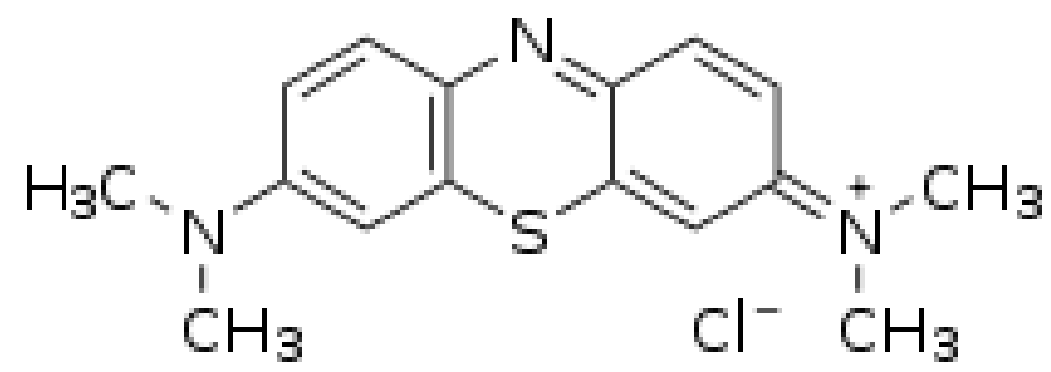

Fig 1: Structural formular of methylene blue.(Calvero, 2006)

It is used in different fields such as Chemistry, Biology and Medicine. At room temperature it is a dark green odourless solid that yields a blue solution when dissolved in water. The hydrated form has 3 molecules of water per molecule of methylene blue. It has a maximum absorption of light around 670nm, though specifics of absorption depend on factors like protonation, adsorption to other materials and metachromasy i.e. the formation of dimmers and higher -order aggregates depending on concentration and other interactions.(Cenens and Schoonheydt(1988). For this work the maximum absorption was confirmed to be $664 \mathrm{~nm}$ which confirms a $\mathrm{MB}^{+}$ species in solution according to Cenens and Schoonheydt(1988).

\section{Methyl orange(MO)}

Methyl orange(CI.13025); Acid Orange 52; [p-(Dimethylamino)-Phenylazo]benzene sulfonic acid, sodium salt; $\mathrm{C}_{14} \mathrm{H}_{14} \mathrm{~N}_{3} \mathrm{O}_{3} \mathrm{SNa}$. $\mathrm{FW}=327.3 \mathrm{~g} / \mathrm{mol}$. (SIGMA 1991). It is a $\mathrm{pH}$ indicator ( at $\mathrm{pH} 3.0(\mathrm{pink})$ $\mathrm{pH} 4.4($ yellow)). It is a $\mathrm{pH}$ indicator frequently used in titrations because of it's clear and distinct colour change. It is also known to posses mutagenic properties.

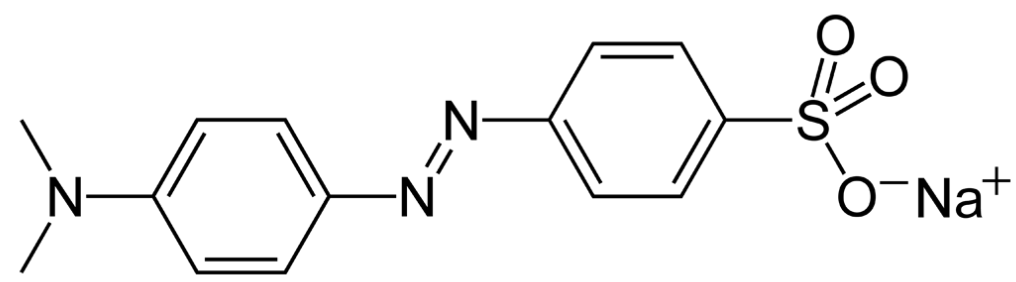

Fig 2: Structural Formular of Methyl orange(Benjah, 2007)

The molecule absorbs blue-green light, which makes it's solution appear red. The nitrogen bearing the positive charge is involved in a double bond.

In the basic form of methyl orange, a hydrogen ion is lost from the $-\mathrm{N}=\mathrm{N}$ - bridge between the rings, and the electrons used to bind the hydrogen neutralize the positive charge on the terminal nitrogen so that it is no longer able to pi-bond. Solutions of MO appear yellow in alkaline solution. The wavelength of maximum absorption of the MO sample used for this work was found to be $464 \mathrm{~nm}$ which agrees with literature.

\section{Indigo carmine (IG)}

Indigo carmine(CI 73015); Acid blue 74; 5,5'-indigosulfonic acid disodium. $\mathrm{C}_{16} \mathrm{H}_{8} \mathrm{~N}_{2} \mathrm{Na}_{2} \mathrm{O}_{8} \mathrm{~S}_{2}$. $\mathrm{FW}=466.3 \mathrm{~g} / \mathrm{mol}$ (SIGMA 1991). It is an approved food colourant in America and the European Union. It is used as a $\mathrm{pH}$ indicator with a blue colour at $\mathrm{pH}$ below 11.4 and yellow at $\mathrm{pH} 13$. It is also used as a dye in capsule manufacturing and in Obstetrics. It's IUPAC name is 3,3'-dioxo-2,2'-bis-indolyden-5,5'-disulfonic acid 
disodium salt. The wavelength of maximum absorption of the IG sample used for this work was found to be $602 \mathrm{~nm}$ which agrees with literature.

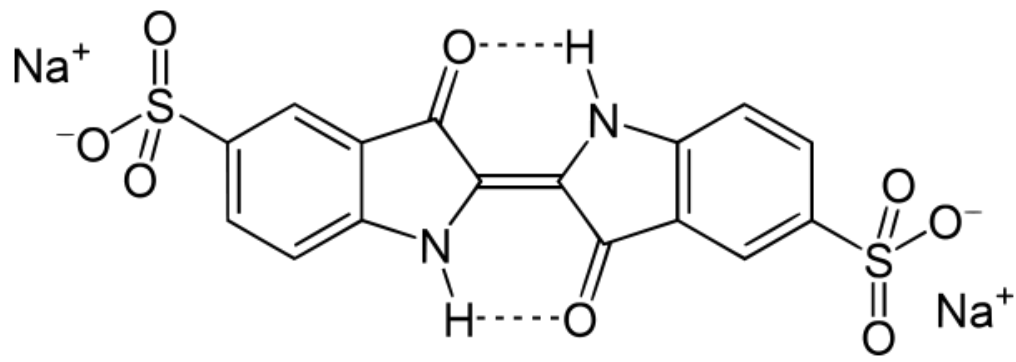

Fig 3: Structure of Indigo carmine.(Yikrazuul, 2009)

\section{Adsorption Studies.}

Adsorption studies was executed using the Batch Mode of interaction. The rate at which the individual dyes (MB, MO and IG) were adsorbed onto UMAC was monitored using two different experiments involving different time frames, AC dose and adsorbate concentration.

In the first arrangement, $10 \mathrm{~cm}^{3}$ of $20 \mathrm{ppm}$ of the individual dyes were separately interacted with $20 \mathrm{mg}$ each of UMAC. Agitation times were for 5, 10, 15, 20, 30, 45, and 60 minutes respectively. The mixtures were allowed to settle and the absorbances of the supernatants were taken using a Spectrophotometer.

In the second arrangement, $10 \mathrm{~cm}^{3}$ of $10 \mathrm{ppm}$ of the individual dyes were separately interacted with $10 \mathrm{mg}$ each of UMAC. This was to observe the adsorption trend when a lower dose of activated carbon was used. Agitation times were for 15, 30, 45 and 60 minutes respectively. The mixtures were allowed to settle and the absorbances of the supernatants were taken using a Jenway Digital UV-Visible Spectrophotometer.

\section{Results And Discussion}

The results obtained from the work were presented using appropriate Tables and Figures while attempts were made to discuss the observations and also state likely inferences based on literature.
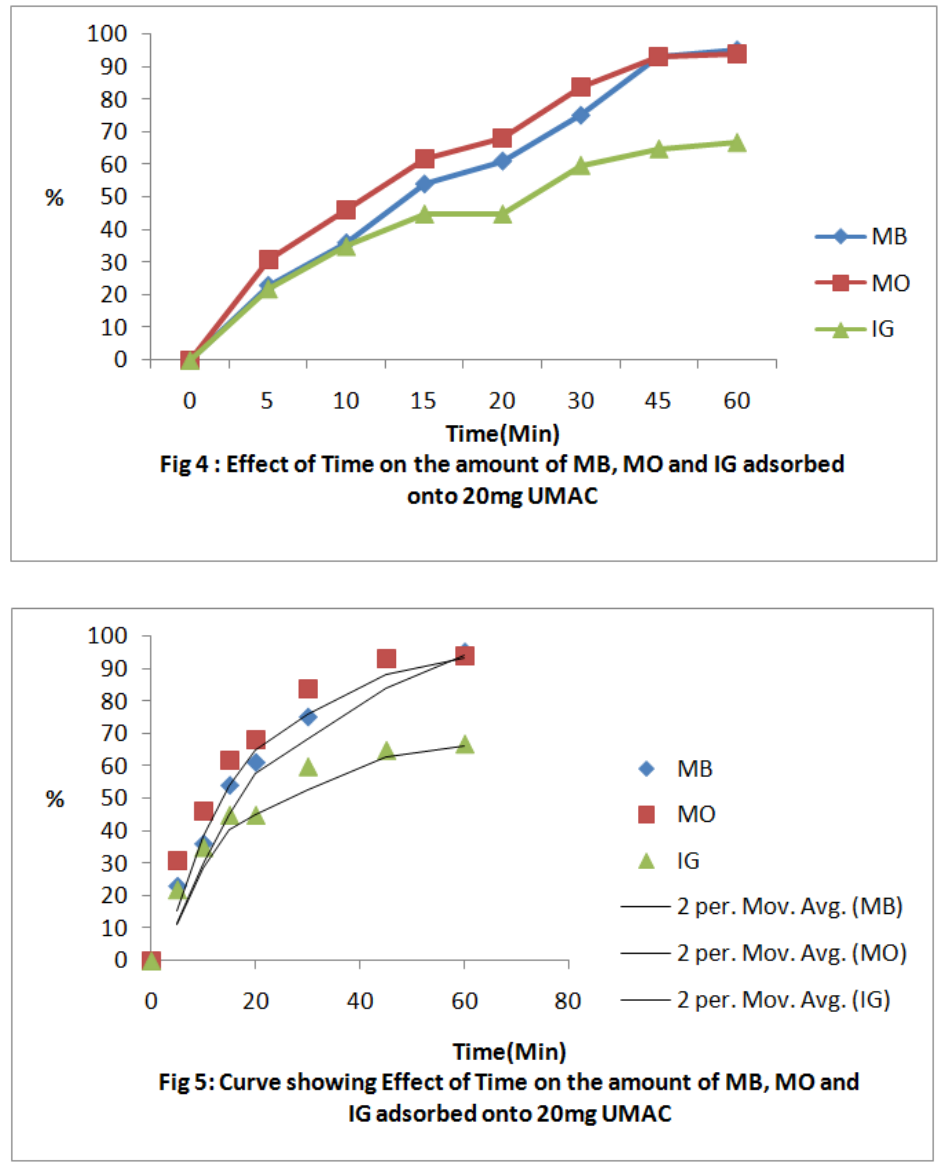

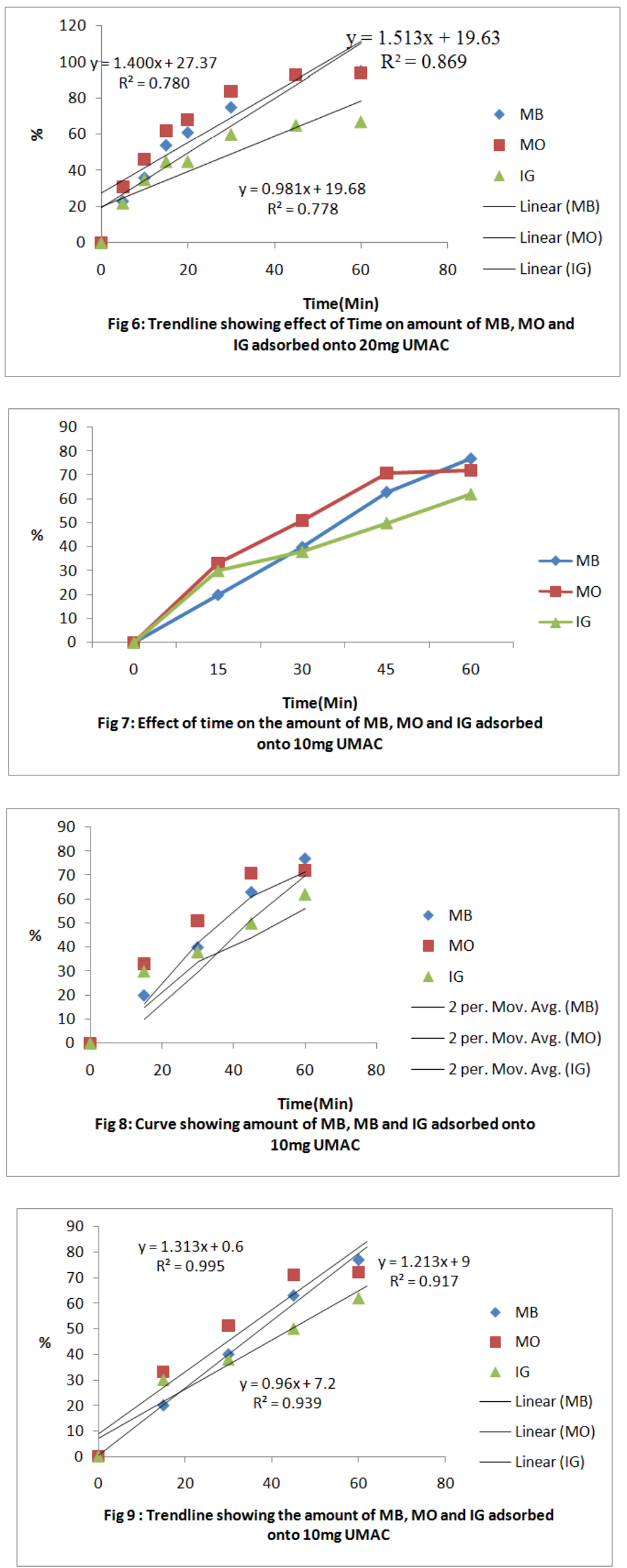

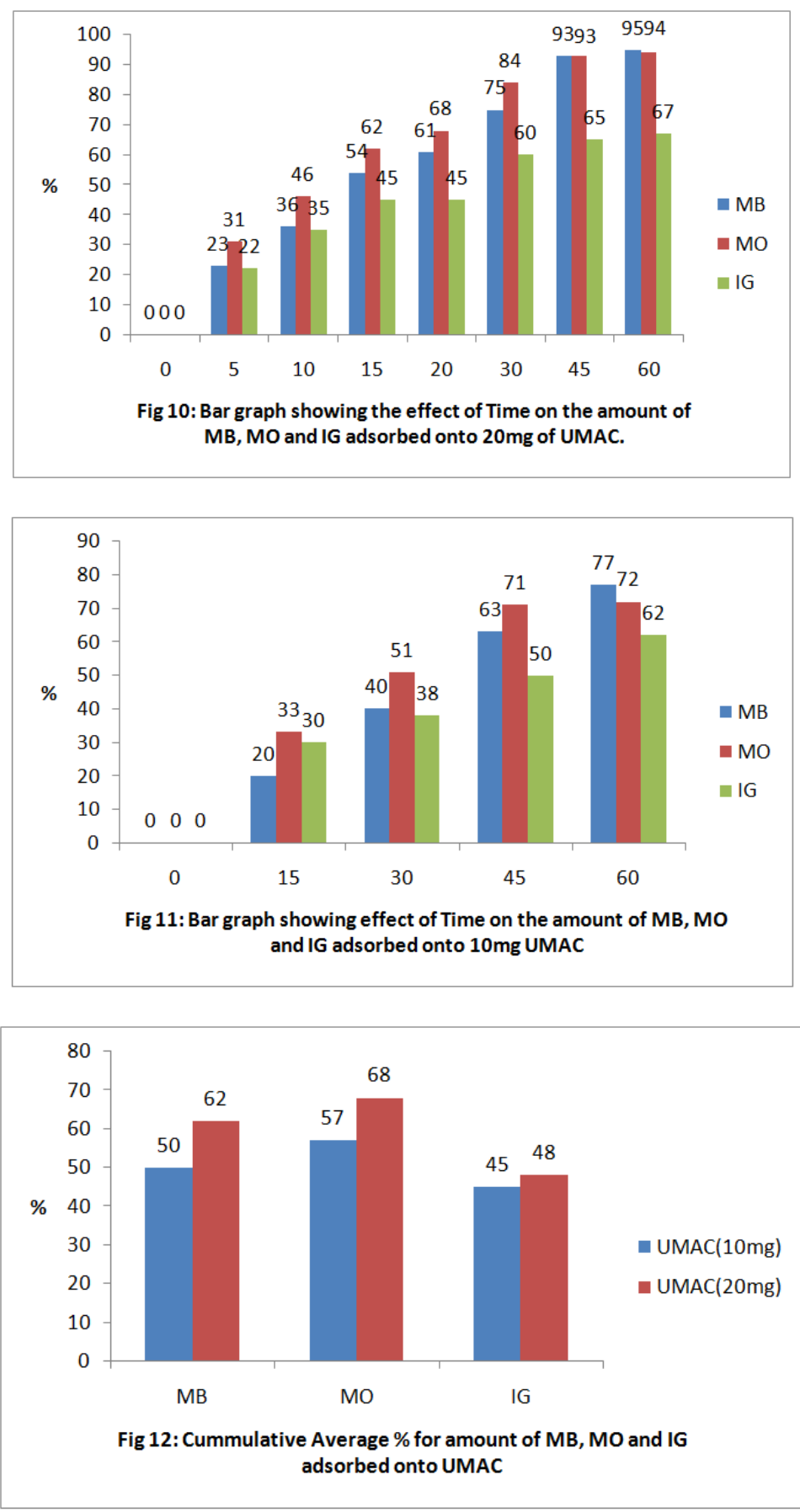

Figure 4, shows the graph for effect of time on the amount of MB, MO and IG individually adsorbed onto 20mgUMAC. Figures 5, 6 and 10 show the graphs for the adsorption curve, adsorption trendline and bar graphs respectively. Fig 4 gives an idea of the sorption process, Figure 5 gives an idea of the movement of the dyes towards adsorption equilibrium states. Simillar curves were reported in works done by Jain et al.(2006), Yasin et al.(2007) and Kathiresan et al.(2010). Figure 6 can be employed for predictive studies, (extrapolation) while Figure 10 gives a general idea of the adsorption trend.

Figures 7, 8 and 9 are similar to Figures 4, 5 and 6 respectively. They convey simillar information. The difference between the Figures 4, 5 and 6 and Figures 7, 8 and 9 is that the adsorption process of the former was based on the interaction of $20 \mathrm{mg}$ of UMAC with $10 \mathrm{~cm}^{3}$ of $20 \mathrm{ppm}$ of the separate dyes and time of 5,10,15, 20, 30,45 and 60 minutes while the process of the latter was based on the interaction of 10mg of UMAC with $10 \mathrm{~cm}^{3}$ of $10 \mathrm{ppm}$ of the separate dyes for 15, 30, 45 and 60 minutes respectively. The results from Figures 4, 5 and 6 reveals more details compared to Figures 7, 8 and 9 . This suggests that changes in dose of adsorbate, concentration of adsorbates and change in agitation periods amplify differences in results. 
From Fig 10, it can be seen that for all the dyes, the amount adsorbed increased as the time increased. But comparatively, MO was adsorbed more than MB and IG, especially in the first 30minutes. MB seems to catch up at 45minutes and then overtakes MO at 60minutes. The amount adsorbed for IG was generally lower than the others. This may be attributed to individual qualities of the dyes like (a) Solubility/Mobility of the dyes (b) Film or Particule Diffusion (c) Size and Orientation of the dyes (Kipling and Wilson, 1960), and also the surface properties of the UMAC sample.

Fig 12 shows the cumulative average \% of the amount of the dyes adsorbed onto UMAC. It is a summary of results in Fig 10 and Fig 11 . This gives a summary of the amount of the individual dyes adsorbed from the two rate experiments performed. The order is $\mathrm{MO}>\mathrm{MB}>\mathrm{IG}$ for both experiments.

Figures 4 and 7 are line graphs for the two separate rate experiments conducted showing the effect of time on the amount of individual dyes adsorbed. The graphs show similar trends but Fig 4 brings more details. From Fig 4 two distinct stages (or steps) can be seen for all the dyes. The first stage is from 0 to $20 \mathrm{~min}$ and the second stage is from $20 \mathrm{~min}$ to $60 \mathrm{~min}$. the first stage can be attributed to film-diffusion of the dyes from the media to the AC surface while the second stage can be attributed to particle-diffusion within the UMAC sample.(Horsfall Jnr and Spiff,2005).

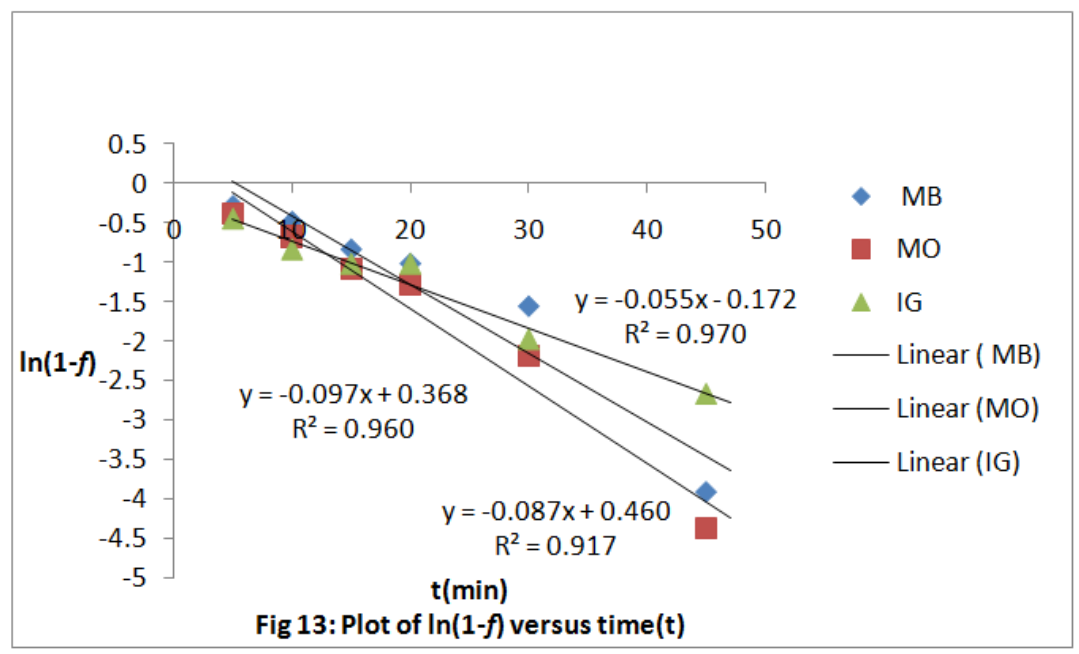

Fig 13 is diffusivity plot of $\ln (1-f)$ against time $(t)$, where $f$ is the fractional attainment of equilibrium which is the ratio of the amount of adsorbates removed from solution after time $(t)$ to that removed when sorption equilibrium is attained.

The theory behind the plot above is that it can be used to explain whether the sorption process is filmdiffusion controlled or particle-diffuson controlled(Ho et al in Horsfall Jnr and Spiff, 2005). If the ln(1-f) against $t$ gives a linear relationship then adsorption is particle-diffusion controlled and the diffusivity of the adsorbate onto the surface is independent of the extent of sorption. However a non-linear plot indicates that the diffusivity of the ions onto the adsorbent surface is controlled by film diffusion( Vinod and Anirudhan in Horsfall Jnr and Spiff; 2005). The plots for the dyes above are all linear with high $\mathrm{R}^{2}$ values. This suggests that for all the dyes involved, the sorption process is particule-diffusion controlled.

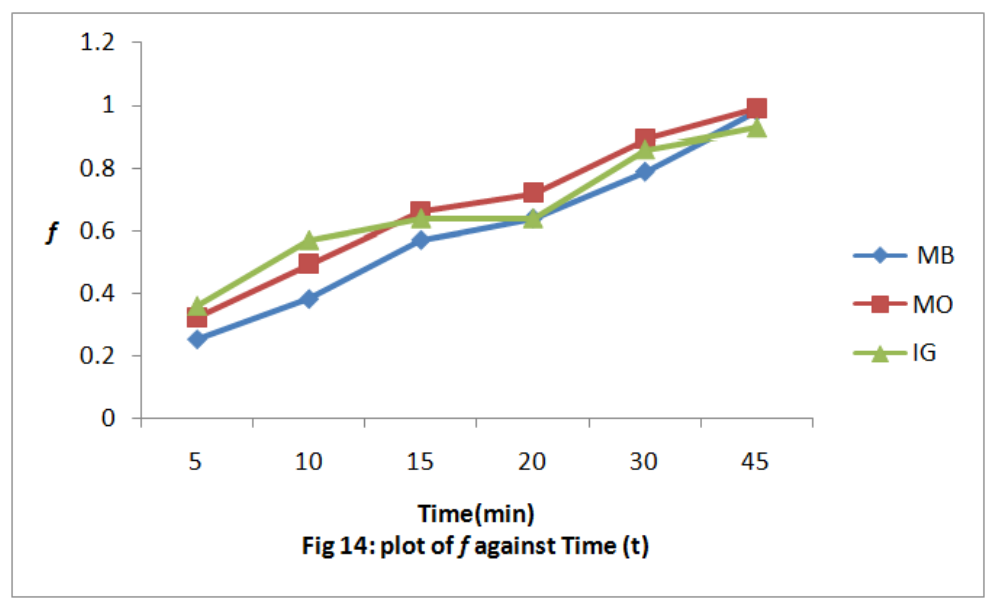


Fig 14 is a plot of $f$ against time $(t)$. It shows an increase(though not linear) in the $\mathrm{f}$ values as contact time (t). This indicates that increasing the contact time may still lead to adsorption. Although, most of the dyes are predominantly taken up within the first 30minutes. This is evident from Fig 5 which shows how the graphs for the dyes curve to attain equilibrium after 30 minutes.

The time taken for adsorbates to reach maximum adsorption is indicative of the type of adsorption occurring. When a long constsant time is needed to attain equilibrium, physisorption may be responsible. A relatively short contact time indicates chemisorptions (Ho et al in Horsfall Jnr and Spiff;2005). Hence, for the two rate experiments performed, chemsorption may be predominant even though physisorption may also be occurring alongside.

Figures 6 and 9 are the trendlines for the two rate experiments. The slope and $\mathrm{R}^{2}$ values for $\mathrm{MB}$ are higher than the MO and IG. This suggests that MB is the most dependent on time for it's adsorption process onto UMAC.

\subsubsection{Influence of size of dyes on adsorption rates.}

For atoms, ions and simple molecules, it is easy to determine parameters like the atomic and ionic radii which give information about their size. For large molecules like dyes, this is not the case. Hence, their sizes may only be predicted. The size or shape of a molecule to an extent will determine the film-diffusion in the medium and particle diffusion within the pore network of the adsorbent(Horsfall Jnr and Spiff, 2005). where the predominant pore size of the adsorbent corresponds with the size of an adsorbate molecule, the adsorption process will be easier and faster; provided all other factors remain constant. For the rate experiments conducted, the adsorbent was constant i.e. UMAC, with a $\mathrm{pH}$ of 7 , the medium was constant i.e aqueous media, the $\mathrm{pH}$ of the aqueous dyes are relatively the same i.e. between $(6.39-6.76)$, this leaves two factors which can influence the adsorption rates. They are (a) the functional groups of the dyes and (b) the size of the dyes. The functional groups of the dyes can be seen from their structures in Figures 1, 2 and 3. Two of the dyes (MO and IG) have similar functional groups, which is the sulphonate group $\left(\mathrm{SO}_{3}{ }^{-}\right)$. If the rates where determined by the functional groups then MO and IG should have been expected to have close rates, yet this is not the case. These narrows the adsorption rates to one factor i.e. the individual sizes of the dye molecules.

Considering the formular weights of the dyes

$\mathrm{MB}=319.9 \mathrm{~g} / \mathrm{mol}$

$\mathrm{MO}=327.3 \mathrm{~g} / \mathrm{mol}$ and

$\mathrm{IG}=466.3 \mathrm{~g} / \mathrm{mol}$.

MB with the smallest weight would have had better rates than MO and IG. The order of the rate of Adsorption on the grounds of their formular weights would have been MB > MO > IG, but the experimental data shows $\mathrm{MO}>\mathrm{MB}>\mathrm{IG}$. However, on looking closely at the dye structures with regards their ring structures and orientation, MO has one ring (benzene) on both sides of the azo group giving a total of two rings. It's structure is symmetrical and linear compared to MB and IG structures. It will also be more flexible than the others.

MB on the other hand has three rings i.e. two benzene rings and one heterocyclic compound. The molecule is symmetrical around the heterocyclic ring. This will restrict the flexibility of the molecule. The molecule is more bulky than MO .

IG has four rings. Two benzene rings and two heterocyclic rings which are bridged by a $\mathrm{C}=\mathrm{C}$ bond and two supporting Hydrogen bonds. The molecule is symmetrical around the conjoined heterocyclics. This will make this molecule the bulkiest of the three and the least flexible or the most restricted in space. From this discussion a trend has evolved which shows a similarity between the dye molecules. The similarity is that :

i. all the molecules are symmetrical in a way.

ii. all the molecules have "a unique bridge."

iii. all the molecules have one benzene ring on both sides of the bridge.

From the above observation and discussion, it can also be seen that the dye molecules also differ with respect to the number of cyclic compounds they are comprised of.

i. MO has two(2) cyclic compounds(i.e. 2 benzene rings),

ii. $\quad$ MB has three (3) cyclic compounds( 2 benzene rings and 1 heterocyclic)

iii. IG has four (4) cyclic compounds ( 2 benzene rings and 2 heterocyclics). The dye molecules have similarities in their differences and differences in their similarities. Based on the number of cyclic compounds, the order of the dyes based on their sizes is represented as below

$$
\mathrm{MO}<\mathrm{MB}<\mathrm{IG}
$$

It can be said for the rate experiment in question, that there is an inverse relationship between the size of the dye molecules and their adsorption rates from aqueous media. The number of cyclic compounds determine the size and bulkiness of the molecules and this is strongly perceived to be the main reason for the 
differences in the adsorption rates of each dye onto UMAC. Where MO being the smallest has the greatest advantage while IG being the bulkiest has the least.

\section{Statistical Analysis of Rate Experiment Results}

Statistical Analysis of the results was done using Sigma Plot 11.0 statistical package; where the \% of the dyes adsorbed as contact time increased was used. These gave three different groups which were subjected to a One way anova test. This gave the mean and standard deviations for each group as follows:

$\mathrm{MB}=62.143 \pm 27.65$

$\mathrm{MO}=68.286 \pm 23.963$ and

$\mathrm{IG}=48.429 \pm 16.612$.

Even though the standard deviations were quite large for each group, the difference in the mean values are not great enough to exclude the possibility that the difference is due to random sampling and that there is no statistically significant difference. The mean values obtained from the test above were then subjected to a OneSample t-test. The result showed that there is a statistically significant difference between the sampled population and the hypothesized population mean $(\mathrm{p}=0.010)$ at $95 \%$ confidence interval.

\section{Conclusion}

From results and discussion of the experiments conducted to monitor the effect of time on the amount of MB, MO and IG adsorbed onto UMAC, the following inferences can be drawn

i. the order for the rate of the amount of the separate dyes adsorbed onto UMAC is

$\mathrm{MO}>\mathrm{MB}>\mathrm{IG}$.

ii. The factor that is strongly responsible for the order in (i) above is strongly attributed to steric factors i.e. size (i.e. bulkiness and orientation) of the individual dye molecules.

iii. the sorption process of the dyes is predominantly Chemisorption

iv. the adsorption process is particle-diffusion controlled

v. MB is the most dependent on time to attain adsorption equilibrium. These results are limited to the aqueous single dye solutions when these dyes are mixed into binary or tertiary systems the preferential adsorption results may not be in the order seen in this case as other factors may come into play.

\section{Acknowledgment}

The researcher wishes to thank the University Board for Research (UBR), Ahmadu Bello UniversityZaria, for partly funding the work

\section{References}

[1]. Benjah-bmm27 (2007). File: Methyl-orange-2D-Skeletal.png. From Wikipedia, the free encyclopedia. Accessed $29^{\text {th }}$ October, 2012

[2]. Calvero (2006). File: Methylene blue.svg. From Wikipedia, the free encyclopedia. Accessed $29^{\text {th }}$ October, 2012

[3]. Cenens, J and Schoonheydt, R.A. (1988). Visible Spectroscopy of methylene blue on Hectorite, Laponite B and Barasym in aqueous suspension. Clay and Clay minerals. 36(3); 214-224 in Wikipedia 2012.

[4]. Hameed, B.H., Mahmoud, D.K. and Ahmad, A.L.(2008). J. Hazard. Mater. 158:499-506 in Vinoth M., Lim, H.Y., Xavier,R., Marimuthu, K., Sreeramanan, S., Mas Rosemal, H.M.H. and Kathiresan, S*. (2010) Removal of methyl orange from solutuions using yam leaf fibres. Int. J. Chem. Tech. Res. 2(4) pp.1892-1900.

[5]. Ho, S.Y., Wase, D.A.J. and Forster, C.F. Water Res. 1995,29,1327 in Horsfall Jnr, M. and Spiff, A.I.(2005). Kinetics of the sorption of $\mathrm{Pb}^{2+}$ and $\mathrm{Cd}^{2+}$ from aqueous solutions by C. bicolor biomass. Bull. Chem. Soc. Ethiop.19(1) pp 88-102.

[6]. Horsfall Jnr, M. and Spiff, A.I.(2005). Kinetics of the sorption of $\mathrm{Pb}^{2+}$ and $\mathrm{Cd}^{2+}$ from aqueous solutions by $C$. bicolor biomass. Bull. Chem. Soc. Ethiop.19(1) pp 88-102

[7]. Jain, R*, Mathur, M. and Sikarwar, S. (2006). Removal of indigocarmine from industrial effluents using iow cost adsorbent. Jour. of Sci. and Indus. Resch. Vol.65 pp 258-263

[8]. Kipling, J.J. and Wilson, R.B.(1960). Adsorption of methylene blue in the determination of surface arears. J. Appl. Chem. 10: 109113.

[9]. SIGMA(1991). Biochemicals, Organic Compounds for Research and Diagonistic Reagents. SIGMA Chemical Company.

[10]. Vinod, V.P. and Anirudhan, T.S. (2001). J. Chem. Technol. Biotechnol. In Horsfall Jnr, M. and Spiff, A.I.(2005). Kinetics of the sorption of $\mathrm{Pb}^{2+}$ and $\quad \mathrm{Cd}^{2+}$ from aqueous solutions by C. bicolor biomass. Bull. Chem. Soc. Ethiop.19(1) pp 88-102

[11]. Vinoth M., Lim, H.Y., Xavier,R., Marimuthu, K., Sreeramanan, S., Mas Rosemal, H.M.H. and Kathiresan, S*. (2010) Removal of methyl orange from solutuions using yam leaf fibres. Int. J. Chem. Tech. Res. 2(4) pp.1892-1900.

[12]. Yasin, Y., Hussein, M. Z. and Ahmad, F.Hj (2007). Adsorption of methylene blue onto treated activated carbon. The Malaysian Jour. of Anal. Sci. Vol11(11):400-406.

[13]. Yikrazuul (2009). File: Indigo carmine.svg. From Wikipedia, the free encyclopedia. Accessed $29^{\text {th }}$ October, 2012. 